\title{
Redes de coautoría como herramienta de evaluación de la producción científica de los grupos de investigación
}

\author{
Julieth Katherine Rodríguez Gutiérrez'; Nubia Yaneth Gómez Velasco²
}

Recibido: 1 mayo 2017 / Aceptado: 31 de octubre de 2017

Resumen. Muestra la importancia del uso de indicadores relacionales en los estudios de análisis de la producción científica de grupos de investigación, con el fin de determinar sus dinámicas de publicación, haciendo énfasis en medidas como la centralidad, la densidad y el tamaño de la red. Se presenta una red de coautoría que visualiza la colaboración entre grupos y las dinámicas de colaboración dentro de cada grupo. Así mismo, se ejemplifica con un caso de estudio, el cálculo de indicadores de la red y su relación con indicadores de productividad. La muestra analizada corresponde a los artículos científicos reportados por 8 grupos de investigación del área de Biología de una Institución de Educación Superior en Colombia, publicaciones vinculadas a los GrupLac de la plataforma ScienTI por cada grupo de investigación. Para elaborar y visualizar la red se utilizó el software Pajek. Se encontró que los grupos de investigación analizados presentan, en general, baja densidad, es decir, un bajo número de vínculos o relaciones entre los autores, igualmente un bajo vínculo entre los grupos de la misma institución en el área de estudio.

Palabras clave: Indicadores relacionales; Redes de coautoría; Grupos de investigación; Producción científica.

\section{[en] Co-authorship networks as a tool for evaluating the scientific production of research groups}

\begin{abstract}
It shows the importance of the use of relational indicators in studies of the scientific production of research groups, in order to determine their publication dynamics, with emphasis on measures such as centrality, density and size of the network. A co-authorship network is presented that allows visualizing the collaboration between groups and the dynamics of collaboration within each group. Likewise, it is exemplified with a case study, the calculation of indicators of the network and its relation with indicators of productivity. The sample analyzed corresponds to the scientific articles reported by 8 research groups in the Biology area of a Higher Education Institution in Colombia, publications linked to the GrupLac of the ScienTI platform by each research group. The Pajek software used to elaborate and visualize the network. It found that the research groups analyzed have, in general, low density, that is, a low number of links or relationships between the authors, also a low link between the groups of the same institution in the study area.

Keywords: Relational indicators; Co-authorship networks; Research groups; Scientific production.

1 Universidad Pedagógica y Tecnológica de Colombia; Grupo de investigación en Estadística GAMMA E-mail: juliethkatherine.rodriguez@uptc.edu.co

2 Universidad Pedagógica y Tecnológica de Colombia; Grupo de investigación en Estadística GAMMA.

E-mail: Nubia.gomez@uptc.edu.co
\end{abstract}


Sumario. 1. Introducción. 2. Metodología. 3. Resultados y Discusión. 4. Conclusiones. 5. Bibliografía.

Cómo citar: Rodríguez Gutiérrez, J. K., Gómez Velasco, N. Y. (2017) Redes de coautoría como herramienta de evaluación de la producción científica de los grupos de investigación, en Revista General de Información y Documentación 27 (2), 279-297.

\section{Introducción}

Los estudios sobre la producción científica han tenido creciente interés para la comunidad científica, ya que permiten conocer el impacto de esta en la sociedad y redirigir los financiamientos a la investigación. Para conocer los aspectos cuantitativos de la investigación se recurre a métodos matemáticos o estadísticos, así como a herramientas útiles, como son los indicadores bibliométricos.

En la evaluación de la producción científica de los docentes y grupos de investigación en instituciones y universidades se sugiere aplicar un sistema de indicadores tanto cuantitativos como cualitativos, que involucren elementos tales como: número de publicaciones, tipo de revista donde se publica, citación $\mathrm{y}$ colaboración (Ospina, 2009); estos dos últimos elementos permiten estudiar con mayor detalle los patrones de citación, para realizar un seguimiento de influencias y redes informales del conocimiento, determinar la amplitud del impacto de la comunidad investigadora y establecer la colaboración entre ellos, dado que las relaciones que el grupo establezca con otros grupos a nivel local, nacional o internacional es un indicio de su dinámica.

Los indicadores bibliométricos permiten realizar una evaluación de la actividad científica, medida a través de las publicaciones, logrando establecer cuatro categorías básicas: quiénes producen (personas, instituciones, países), cuánto producen (cantidad de documentos), qué tan citado es lo producido y cómo colaboran los grupos de investigación o instituciones (redes) (Bordons y Zulueta, 1999).

Los indicadores bibliométricos se pueden clasificar en: unidimensionales, que usan técnicas estadísticas univariadas, y multidimensionales o relacionales, basados en técnicas estadísticas multivariadas que estudian varias características de los documentos analizados y establecen múltiples interrelaciones en las publicaciones o en los hábitos de investigación de los científicos (Sanz Casado, 2000). Estas múltiples relaciones pueden representarse gráficamente a través de mapas bibliométricos (Tijssen y van Raan, 1994; Trujillo et al., 2013) o a través del análisis de redes (Abbasi y Altmann, 2010).

Adicionalmente, los indicadores multidimensionales o relacionales son asociados a técnicas de mapeo, en alusión a los gráficos que se generan y que comúnmente se han llamado "mapas" (Spinak, 1996). El análisis estadístico que subyace en la elaboración de estos mapas se basa en el análisis de matrices de datos multivariados. Al trabajar con una matriz de contingencia, una técnica de análisis es a través de las redes sociales, que visualizan las relaciones que se establecen entre grupos de investigación e instituciones, en torno a las publicaciones. 
El objetivo del artículo es mostrar la importancia de los indicadores relacionales, medidos a través de redes de coautoría, y del uso de indicadores de la red, usando medidas de centralidad, densidad y tamaño de la red. Estos indicadores serán ejemplificados en detalle, de forma que facilite su comprensión y obtención, potencializando así su uso en otros estudios relacionados con la producción científica y las redes de coautoría.

\subsection{Mapas para los indicadores relacionales}

Dependiendo de las variables que se analicen, pueden obtenerse varios tipos de representaciones, descritos por Sanz y Martín (1997) como: Redes cognitivas, que agrupa a los autores por temáticas e identifica las líneas de investigación más trabajadas; permite determinar las relaciones existentes entre las distintas disciplinas científicas, e identificar y evaluar el núcleo de revistas científicas dedicadas a una determinada disciplina. Mapas temáticos, lo que permite realizar el análisis de co-palabras, es decir, aparición conjunta en los documentos de los mismos descriptores, palabras clave o determinadas palabras del título de las publicaciones. Redes de colaboración, el cual determina cuáles autores producen, cuánto producen, con quiénes escriben y cómo se colaboran.

Las representaciones de redes de colaboración son de gran importancia, ya que permiten a los financiadores ubicar a los grupos o instituciones que más producen, para considerarlos al asignar mayor financiamiento, así como conocer cuáles son las redes de colaboración, que estimulen o generen nuevas áreas de colaboración (Huamaní \& Mayta, 2010). Según Sanz Casado (2000), la colaboración entre grupos tiene un efecto positivo sobre la producción científica, tanto cuantitativa como cualitativa, en especial cuando ésta se lleva a cabo por comunidades científicas y grupos del más alto nivel.

Se ha demostrado que las investigaciones que se realizan en equipo son más productivas y adquieren mayor número de citaciones que cuando se trabaja en forma aislada o con escasa colaboración. Esta dinámica de generación de conocimiento se puede evaluar a través de redes de colaboración, que llegan a ser un reflejo de las relaciones dadas entre grupos, quienes pueden unir instituciones a través de sus vínculos laborales y académicos.

\subsection{Redes sociales o redes de coautoría}

De acuerdo con Almero (2011), el Análisis de Redes Sociales (ARS) representa una revolución en los estudios bibliométricos sobre la actividad científica, ya que permite estudiar las relaciones entre los elementos de un entorno determinado; así, los estudios bibliométricos se interesan tanto por la producción científica de las instituciones, como por las redes de colaboración que puedan existir entre ellas. De esta forma, las redes, han supuesto una mayor contribución al nivel de análisis de la investigación científica, permitiendo el estudio de grupos de investigación como unidad experimental, incluso al tratar gran volumen de información, como puede ser la producción científica nacional. Estos elementos son reforzados desde la 
mirada de Schultz-Jones (2009), al indicar que realizando un gráfico de la estructura de las interacciones, un investigador puede identificar los canales a través de los cuales la investigación fluye de un nodo a otro, así como la posible influencia de uno sobre otro.

De acuerdo con lo expuesto por Almero (2011), el ARS permite definir diferentes elementos (tamaño, estructura y composición) en los grupos de investigación, los cuales pueden influir en el rendimiento y la eficiencia de ellos; esta propiedad podría permitir a los responsables de políticas científicas diseñar iniciativas que fomenten un comportamiento más eficiente de los grupos. Elementos que así mismo son considerados por González y Gómez (2014) quienes sostienen que estudios sobre colaboración científica proporcionan a los miembros de la comunidad investigadora y a los responsables de las políticas científicas, evidencias objetivas para la toma de decisiones; reconociendo a su vez, que el fenómeno de la colaboración científica es complejo y diverso, lo que requiere profundizar en líneas de investigación encaminadas a identificar diversas formas de colaboración establecidas por la comunidad.

Según Fonseca et al. (2007), las redes permiten realizar un acercamiento a la dinámica que se genera dentro de las investigaciones científicas, y con los resultados de las investigaciones que se plasman en artículos, es posible realizar análisis de redes de coautoría, cuyos nodos son los autores, generándose un vínculo entre dos nodos, cuando dos investigadores aparecen en una misma publicación. Así, es posible usar la bibliometría, complementada por técnicas de ARS, para estudiar patrones de coautoría entre los integrantes de una comunidad científica, y proporcionar una imagen de los grupos de investigación en una disciplina, su distribución, su importancia y sus interacciones o la falta de ellas.

\subsection{Ventajas del uso de redes de coautoría}

Velden, Haque \& Lagoze (2010) indican que mediante las redes sociales que se construyen a partir de la coautoría en las publicaciones científicas, se pueden tomar los grupos de autores como huellas bibliométricas para medir la producción colectiva de conocimiento.

Existen diversas razones para el análisis de las redes de coautorías. Lassi y Sonnenwald (2010) hacen referencia a diferentes ventajas para la colaboración científica, entre las que se puede destacar: los trabajos con varios autores son, con frecuencia, más citados y durante mayor tiempo; los organismos financiadores de investigación fomentan la investigación en colaboración; la colaboración incrementa la eficiencia, debido al mejor uso de los recursos existentes; los grupos de investigación grandes pueden abordar grandes proyectos; el trabajo en colaboración permite la transferencia del aprendizaje, y, ser miembro de un grupo de investigación aporta prestigio.

Troyano et al. (2005) indican que por medio de las redes de coautorías es posible analizar una de las estructuras más importantes que componen una comunidad científica: su red social; esto es posible gracias a que la red de coautoría, en gran medida, refleja las relaciones sociales que existen entre los 
miembros de una comunidad. Por su parte, Vargas y Castellanos (2005) mencionan que, mientras los indicadores de actividad proporcionan datos sobre el volumen y el impacto de las actividades de investigación mediante sencillos recuentos de elementos bibliográficos, los indicadores relacionales se proponen conocer las interacciones entre estos elementos, intentando describir el contenido de las actividades y su evolución. Algunos autores sugieren que los indicadores relacionales intentan caracterizar los modos de relación y organización de los científicos, ya sea en forma de coautorías, en grupos estables de investigación o en redes académicas formales e informales.

En este sentido, las redes se consideran un indicador relacional cuyos elementos básicos son los actores y las relaciones. Los actores, también denominados nodos, hacen referencia a cada uno de los elementos que componen la red, y son representados mediante puntos; las relaciones son las encargadas de conectar a unos actores con otros, y se visualizan mediante líneas. Es de precisar, que el ARS proporciona sus propios indicadores, como el tamaño de red, la densidad de la red y la centralización de grado de la red, obtenidos a partir de la estructura de las redes y la posición de los nodos, los cuales completan y complementan los indicadores bibliométricos más extendidos en política científica, como son la productividad y el impacto (Sharma y Urs, 2008).

Si bien la colaboración científica y las redes, generas algunas ventajas en las dinámicas de publicación que se realizan en las instituciones públicas como es el caso de las universidades, igualmente se han dado algunos debates sobre los aspectos negativos o cuestionables en torno a lo que de ahí se desprende. Leahey (2016) presenta algunas reflexiones respecto a los costos de la colaboración que pueden generarse al no contar entre sus miembros con un lenguaje común en el abordaje de la problemática, las dificultades por los límites disciplinarios o institucionales, la no alineación de los objetivos del equipo de trabajo, entre otros; aspectos a tener presente en la clasificación de los tipos de colaboración y los impactos esperados.

\subsection{Antecedentes de estudios publicados sobre temas afines}

En Corral y Cànoves (2014) se expone tres niveles de organización, la unidad micro, que la constituyen los grupos de investigación y equipos de trabajo; la unidad meso, que son los institutos y departamentos, y macro, correspondiente a universidades y ministerios.

Para Rey et al (2008), un grupo de investigación es un conjunto de dos o más personas que desarrollan su actividad científica en la misma unidad (departamento, laboratorio, etc.), con líneas de investigación, objetivos e intereses científicos comunes; que comparten tareas y recursos, publican conjuntamente de forma habitual, y disponen de cierta autonomía económica y de decisión. Por su parte, Colciencias (entidad encargada de la medición y categorización de los grupos de investigación científica y tecnológica en Colombia) en la convocatoria a la creatividad de 1992, definió 'grupo de investigación' como el núcleo o unidad básica del Sistema Nacional de Ciencia Tecnología e Innovación-SNCTeI, para la 
generación de conocimiento a partir de la investigación. De igual forma, Lee et al. (2011) y Sorensen et al. (2010), concuerdan que el grupo de investigación es la unidad de producción del conocimiento en las mayorías de las comunidades académicas y científicas, y que las redes de coautoría permiten estudiarlos, analizarlos y visualizarlos en términos de colaboración científica.

En Colombia son pocos los estudios realizados sobre producción científica y, en particular, son escasos los estudios realizados alrededor de la productividad de los grupos de investigación, que toman como fuente central de información los datos reportados en el GrupLac. Se encuentran estudios como el de García (2013) que analiza las redes de coautoría de 76 grupos de investigación de la Universidad Politécnica de Valencia (México), y el efecto que tiene la estructura de la red de los grupos en su productividad científica, los resultados muestran que existe una relación entre las variables de red y la productividad de los grupos.

Por otra parte, Aguado et al. (2009) ofrecen un análisis de los patrones de colaboración mediante redes de coautoría para cinco revistas editadas por la Universidad Autónoma del Estado de México (UAEM). El análisis les permitió conocer algunas características de la estructura de las redes de colaboración tanto de los autores, como de las instituciones y de los países que participan. Corral y Cànoves (2014) analizan la colaboración científica en el campo del turismo en Cataluña; caracterizan los grupos de investigación; estudian la colaboración de grupos, instituciones y países; exploran el efecto de la multidisciplinariedad de las revistas, e identifican autores clave, mediante el cálculo de indicadores bibliométricos y el análisis de redes sociales.

Se encuentra también el trabajo de Navarro y Salazar (2007), en el que se expone un sistema que toma los currículos de investigadores en ciencia y tecnología de la plataforma chilena SICTI (Sistema de Información en Ciencia, Tecnología e Innovación) para generar archivos de redes sociales y posteriormente analizar redes de investigación en ciencia y tecnología. Perianes-Rodríguez et al. (2007) indican que en estudios de colaboración científica a través de redes de coautoría están siendo aplicados los métodos del análisis de redes, y se consideran las teorías o argumentos provenientes del capital social para analizar las redes de colaboración.

Los principales resultados de Hou et al., (2008) y Van der Leij y Goyal (2011), identifican que los autores más productivos en su disciplina científica ocupan una posición central en la red y son muy activos en la red de colaboración de su disciplina. De acuerdo con Van der Leij y Goyal (2011), la red central de la disciplina está formada solo por un pequeño grupo de investigadores muy productivos, mientras que la mayoría de los autores, con baja productividad, ocupan una posición periférica en la red.

\section{Metodología}

Para los estudios de producción científica es frecuente que se utilice la información reportada en grandes bases de datos, como ISI o Scopus; sin embargo, a la hora de analizar la productividad de los grupos de investigación es recomendable utilizar 
otras fuentes de información, como los Sistemas de Información de Ciencia y Tecnología implementados en algunos países.

En Colombia se cuenta con una base de datos completa sobre producción científica y académica, en la que es posible identificar a los actores que intervienen en los procesos de investigación y generación de nuevo conocimiento (Plataforma ScienTI de Colciencias); en ella se permite el registro en línea, a partir de sistemas de acumulación y procesamiento de información, de las actividades de la comunidad científica y tecnológica nacional, lo que contribuye con la definición de políticas, estrategias y mecanismos que apoyan la consolidación de capacidades científicas en el país. Esta plataforma es alimentada por las instituciones InstituLac y la comunidad científica que diligencia el CvLac, y a través de estos se reportan las actividades a los grupos de investigación GrupLac.

En este sentido, dicha política permite identificar y reconocer las dinámicas de los grupos de investigación, con el fin de establecer estrategias para promover el fortalecimiento y consolidación de estos, así como la creación de nuevos grupos. Sin embargo, al emplear la información reportada en los Sistemas de Información de Ciencia y Tecnología se deben tener en cuenta algunas limitaciones de la información:

1) Se confía en lo que está escrito: toda la información del currículo es ingresada por el usuario. No se puede verificar la validez de la información o posibles errores.

2) Información omitida: no siempre el usuario provee toda la información que se desea analizar, lo que hace que en algunos campos haya poca información.

3) No hay identificador único: el nombre de una entidad puede estar escrito de distintas formas, lo que hace que se requiera procesamiento adicional para identificarla correctamente.

4) Nombres repetidos: no hay una forma única de identificar.

Como caso de estudio, en el presente trabajo se analizó la productividad de grupos de investigación durante el periodo 2001-2014, tomando como disciplina el área de Biología, de la Universidad Pedagógica y Tecnológica de Colombia (Uptc). La información de la productividad que se analizó fue la encontrada en la plataforma ScienTI, directamente de lo reportado en los GrupLac por los integrantes de los ocho grupos de investigación activos en el momento de la recolección de la información. Se analizó únicamente lo correspondiente a la productividad de artículos científicos, teniendo en cuenta que son los que mayor visibilidad e impacto tienen en la comunidad científica internacional y nacional.

Con base en la información reportada por los grupos de investigación se calcularon algunos indicadores de redes de coautoría, entre ellos: tamaño de red, densidad y centralidad de grado. 


\subsection{Tamaño de red $(T)$}

El tamaño de red $(\mathrm{T})$ es la propiedad que mide el número de actores o individuos que participan en la red social (Tichy et al., 1979). Cuantos más vínculos o relaciones tenga un autor, mayor será su red, y reflejará mayores recursos y mayor acceso a información y conocimiento. T está determinado por el número total de autores distintos que publicaron en el periodo de estudio.

\subsection{Densidad (D)}

La densidad de la red (D) es la propiedad que mide la proporción de las relaciones presentes en ella sobre el máximo número de relaciones que pueden existir. El número máximo de relaciones está determinado por el número total de nodos que hay en la red. La densidad de la red de cada grupo varía en un rango de 0 a 1 , en donde cero significa que no existe relación entre los integrantes del grupo, y uno significa que todos los integrantes del grupo están relacionados entre ellos, siendo el nivel máximo de relación que puede haber entre los integrantes del grupo (Wasserman y Faust, 1994).

La densidad de la red se minimiza cuando no existen relaciones entre los actores, y se maximiza cuando todos los actores están relacionados entre sí (Reagans y Zuckerman, 2001).

La relación matemática que representa este indicador está dada por:

$$
D=\sum_{i=1}^{N} \frac{C_{G}\left(n_{i}\right)}{N(N-1)}=\frac{\sum_{i=1}^{N} C_{G}\left(n_{i}\right)}{N(N-1)}
$$

Donde $C_{G}\left(n_{i}\right)$ representa la centralidad de grado de cada actor, es decir, el número de relaciones o vínculos en cada nodo. En Freeman (2000), esta medida se obtiene a partir de la expresión

$$
C_{G}\left(n_{i}\right)=\sum_{k=1}^{N} a\left(n_{i}, n_{k}\right)
$$

Donde $a\left(n_{i}, n_{k}\right)=1$ si existe un vínculo entre el autor $n_{i} y n_{k}$, de lo contrario, toma un valor de cero.

\subsection{Centralidad de grado de la red $\left(C_{G}\right)$}

La centralización hace referencia a la centralidad de la red, que identifica los nodos con mayor número de vínculos dentro de una red. A nivel grupal, la propiedad de centralidad de la red se basa en los índices de centralidad de cada uno de los nodos, y se usa para medir el grado en el que la red del grupo está concentrada alrededor de uno o pocos nodos.

Autores como Luo (2005) relacionan la centralidad de la red del grupo con aspectos de liderazgo, al considerar que una red centralizada puede indicar la existencia de un liderazgo fuerte y la posibilidad de que exista mayor control de las 
actividades del grupo y mayor organización; aunque también una red muy centralizada puede limitar la libertad de acción de los integrantes del grupo.

Para medir la centralidad de grado de la red se aplicó la medida de centralidad de grado propuesta por Freeman (2000) y trabajada en Polanco (2006):

$$
C_{G}=\frac{\sum_{i=1}^{N}\left[C_{G}\left(n^{*}\right)-C_{G}\left(n_{i}\right)\right]}{N^{2}-3 N+2}
$$

Donde $C_{G}\left(n^{*}\right)$ es el máximo valor de centralidad de un actor en la red; $N$, el número de autores, y $C_{G}\left(n_{i}\right)$, el grado de centralidad del actor $i$-ésimo.

Dado que $C_{G}$ es la razón de una suma de diferencias observada respecto a su valor máximo, oscilará entre 0 y $1 . C_{G}=0$ indica que las centralidades de cada uno de los nodos son iguales, y $C_{G}=1$ indica, si y solo si, que un nodo de la red la domina completamente en lo que a centralidad se refiere (Freeman, 2000).

Polanco (2006) identifica y define los tipos de grafos con sus relaciones, junto con los tipos de nodos, siendo estos clasificados como: Aislado, Transmisor, Receptor y Portador.

\subsection{Software para el análisis de redes de coautoría}

Existen diversos softwares para el análisis y la visualización de redes, algunos son de libre distribución como Pajek, Publish or perish, VOSviewer y Visone; para otros es necesario adquirir la licencia de uso, como UCINET y Vantage Point. En este estudio se utilizó Pajek, software que ha sido utilizado para el análisis de grandes redes; es gratuito, pero su uso se limita para fines no comerciales.

Para generar una red de coautoría con Pajek, en la que se identifiquen algunos patrones de colaboración (interna, externa, nacional e internacional), productividad de los autores y la relación entre ellos, es necesario crear tres ficheros o archivos. En el primer archivo se especifican cada uno de los autores, quienes representarán cada nodo; además, deben especificarse las relaciones existentes entre los autores (dos a dos) y cuántas veces se han comunicado entre ellos. En un segundo archivo se indica en cuántas publicaciones aparecen cada uno de los autores. Y en el tercer archivo se establecen las agrupaciones que permitirán visualizar los patrones de colaboración.

Una desventaja de utilizar Pajek es que los archivos se elaboran de forma manual y para gran volumen de datos resulta tediosa la elaboración de los ficheros, ya que para algunos es necesario obtener primero una tabla de doble entrada o matriz nxp, y a partir de ella establecer las relaciones existentes.

En el siguiente diagrama se presenta un esquema metodológico de técnicas cienciométricas y bibliométricas que se usan en el análisis de la producción científica, así como de herramientas y softwares que facilitan el análisis y la visualización de las redes de coautoría. Se observa que el análisis de redes, con indicadores como centralidad, densidad y tamaño de la red, se encuentra dentro de los indicadores bibliométricos multidimensionales. 
Gráfico 1. Esquema metodológico de técnicas cienciométricas y bibliométricas

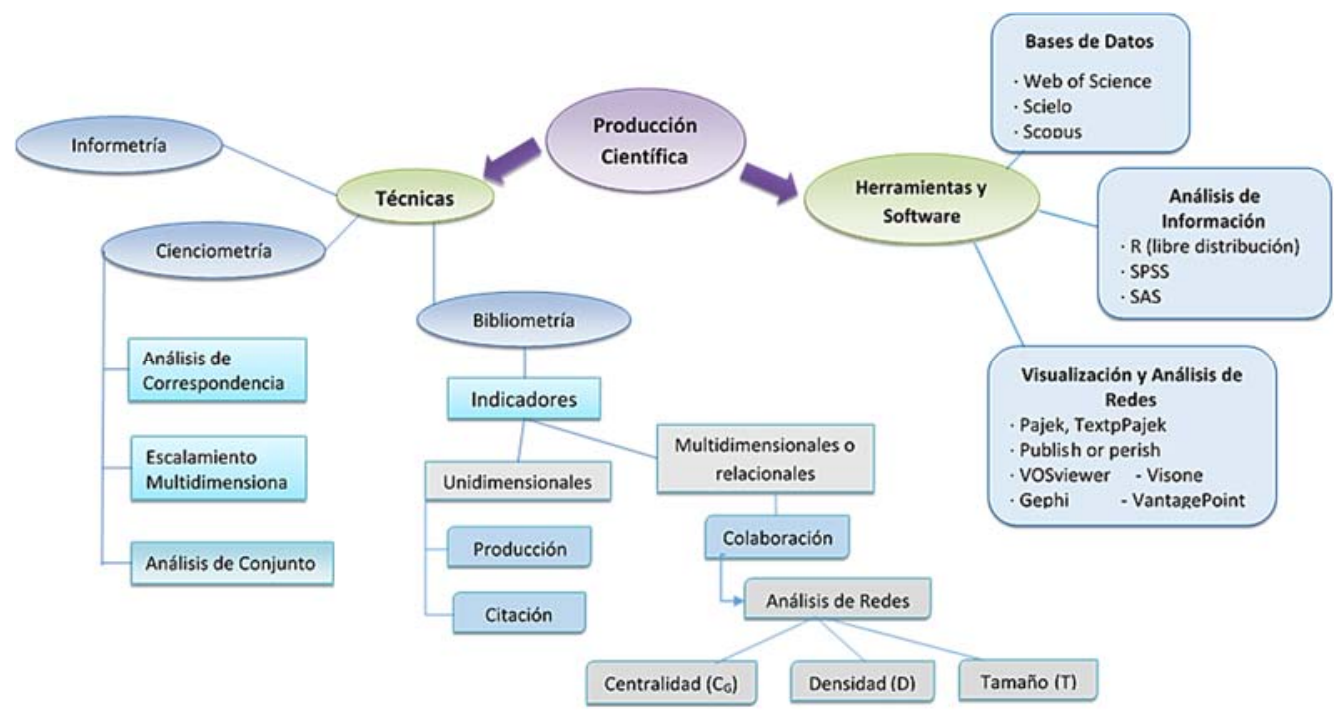

A través del software Pajek se construyó la red de coautoría de todos los grupos objeto de estudio, con el fin de determinar, entre otros aspectos, si los grupos se colaboran entre ellos en sus investigaciones y posteriores publicaciones.

Es de precisar que, el análisis de las redes, se basa en la información reportada por la comunidad investigadora de los grupos de investigación que vinculan al GrupLac, por tanto, se representa a través de las redes de coautoría las dinámicas de publicación de lo que aportan los integrantes a los grupos. Así entonces, se visualiza la red tanto de los integrantes de cada grupo como la colaboración entre los diferentes grupos.

\section{Resultados y discusión}

El estudio se realizó con ocho grupos de investigación del área de Biología, siendo estos: BIOPLASMA (G1), EBAC (G2), GEBIMOL (G3), GEO (G4), GIBC (G5), SISBIO (G6), UDESA (G7) y XIUÄ (G8). Grupos que están conformados entre 11 y 44 investigadores activos, con un promedio de 23 investigadores activos por grupo. La mayoría de estos grupos tienen más de 10 años de creación, y cuentan por lo general con 2 a 5 líneas de investigación, sus líderes en su mayoría cuentan con títulos de doctorado.

La producción total de los ocho grupos activos de investigación del área de Biología, de la UPTC, en el periodo 2001-2014, fue de 162 artículos, que contaron con un total de 540 firmas. El 34,8 \% de los autores reportaron afiliación internacional. En el gráfico 2 se presenta la red de coautoría de los grupos, en la cual se identifican tres patrones de colaboración: afiliación institucional internacional (rojo), afiliación institucional colombiana externa (verde) y afiliación institucional interna (amarillo). Igualmente, se visibiliza cada una de las subredes 
generadas dentro de cada uno de los grupos de investigación (ocho subredes, que corresponden a 8 grupos de investigación institucionalizados y con GrupLac y hoja de vida del grupo visibles en la plataforma red ScienTI).

En la red se visualiza la existencia de subredes con predominancia de vínculos institucionales (Uptc), como es el caso de los grupos G5, G1 y G2; subredes con mayores vínculos interinstitucionales (Uptc y otras instituciones nacionales), como se observa con los grupos G4 y G3, y subredes con alta participación de vínculos internacionales, de los grupos G6 y G8. Las líneas de investigación de cada uno de estos grupos junto con el número de artículos publicados en el período de estudio, y los indicadores de red, se identifican en la tabla 1.

Realizando un análisis con mayor detalle, fue posible establecer que la mayor colaboración interinstitucional se dio con la Universidad Nacional de Colombia y los países con mayor coautoría fueron: Estados Unidos, Costa Rica y Francia. Así mismo, las revistas donde hubo mayor publicación durante el periodo de estudio fueron: Ciencia en Desarrollo y Acta Biológica Colombiana; la primera es editada en la misma universidad a la cual pertenecen los grupos de investigación, y la segunda es una revista nacional, con visibilidad internacional, ubicada dentro de las primeras 25 revistas con mayor publicación en la producción científica nacional (Scopus, 2014).

Gráfico 2. Red de coautoría, investigadores de los grupos de investigación activos en el área de Biología-UPTC, 2001-2014

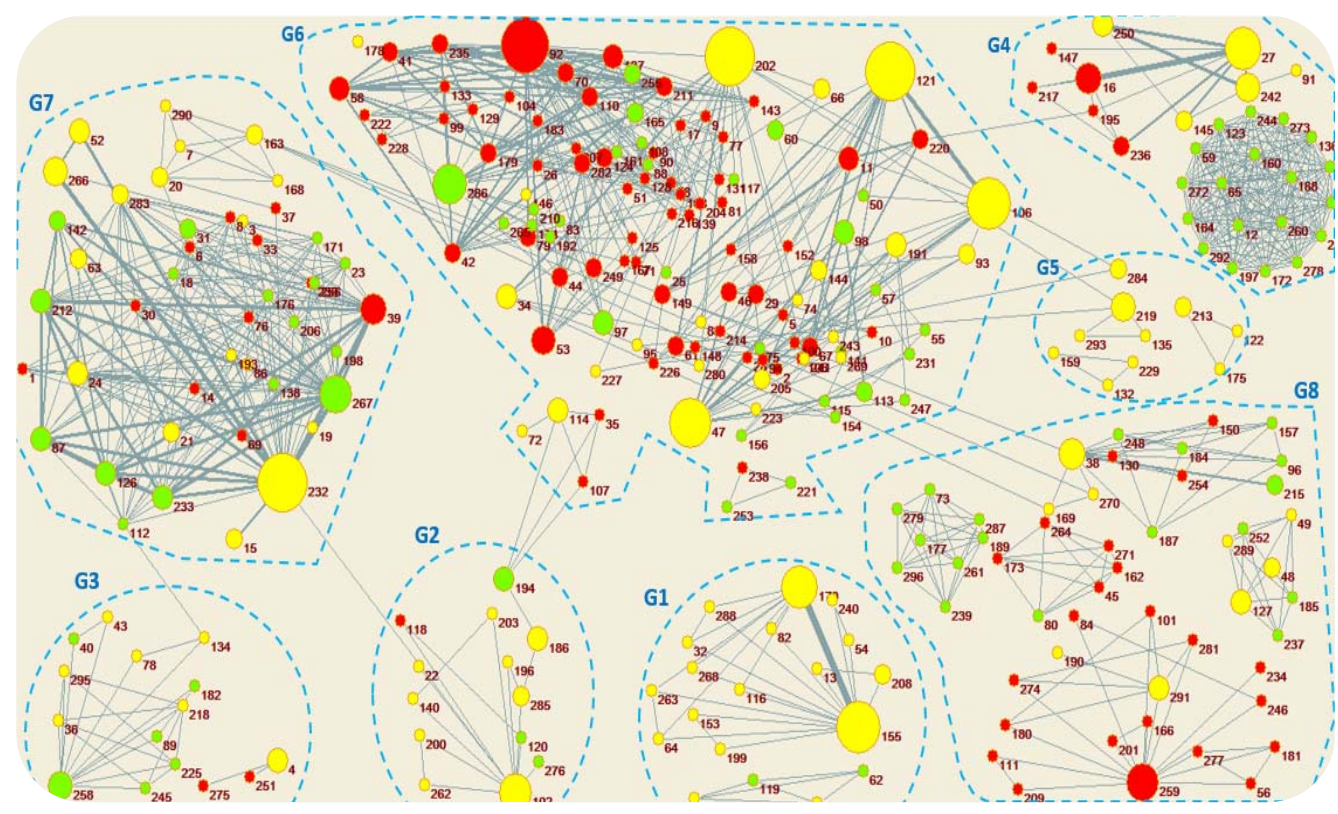

El diámetro de los nodos se relaciona con la productividad de cada uno de los autores; las líneas o vínculos establecen si existe o no relación entre un par de autores; el grosor de las líneas hace referencia a qué tanto se comunican dos o más autores. 
En la red de coautoría del gráfico 2 se identificó que los autores cuyo nodo presentaba mayor diámetro son los más productivos de los grupos de investigación; varios de ellos han actuado como coordinadores de los grupos durante un largo periodo.

Mediante los vínculos (líneas) es posible observar que los autores no publican frecuentemente con los mismos coautores, lo que hace que la red sea menos densa, y que se conformen más equipos de trabajo. Puede observarse, además, que en cada grupo de investigación se busca publicar con autores con afiliación institucional internacional, más que con los de la misma universidad; esto puede deberse a que los trabajos en colaboración internacional son más citados (Narin et al., 1991; Lewison, 1991); es decir, que los autores buscan, mediante la generación de redes científicas con coautores de otras universidades o países, que sus publicaciones tengan mayor visibilidad.

\subsection{Indicadores de la red}

A continuación, se presentan los indicadores descritos en la teoría referida anteriormente, ilustrando el caso para toda la red de coautoría y para cada uno de los grupos de investigación, enfocándose en las medidas de tamaño de la red $(T)$, Densidad (D) y Centralidad de Grado $\left(\mathrm{C}_{\mathrm{G}}\right)$, con el fin de mostrar tanto de forma gráfica como numérica lo que representa cada una de estas medidas.

\subsubsection{Indicadores de la red de coautoría}

Tamaño de red (T). Este indicador hace referencia a la cantidad de nodos presentes en la red, es decir, la cantidad de autores que publicaron en el periodo de estudio, la cual corresponde para este caso a 296 autores.

Densidad de la red (D). Con este indicador se determina la cantidad de relaciones existentes en la red respecto a la cantidad de relaciones posibles. Siguiendo la ecuación 1 , se tiene:

$$
D=\sum_{i=1}^{N=296} \frac{C_{G}\left(n_{i}\right)}{296(296-1)}
$$

La centralidad de grado, $\boldsymbol{C}_{\boldsymbol{G}}\left(\boldsymbol{n}_{\boldsymbol{i}}\right)$, de cada actor o nodo (autor), hace referencia al número de vínculos de cada autor, es decir, la cantidad de relaciones entre un autor y otros. Así, por ejemplo, el autor $1\left(\mathrm{n}_{1}\right)$, que tiene 2 artículos, el primero publicado con un total de 3 autores $\left(\mathrm{n}_{1}, \mathrm{n}_{2}\right.$ y $\left.\mathrm{n}_{3}\right)$ y el segundo con un total de 2 autores $\left(\mathrm{n}_{1}\right.$ y $\mathrm{n}_{2}$ ), generó 3 vínculos, formados como se ilustra en la gráfica. 
Articulo 1

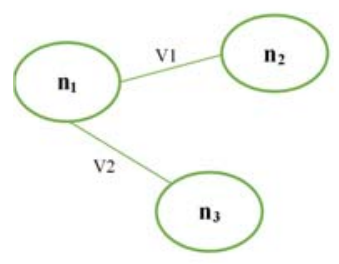

Articulo 2

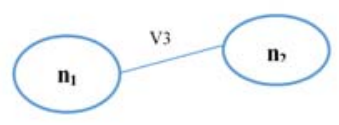

Se observa que en el primer artículo las relaciones correspondientes son: $a\left(n_{1}, n_{2}\right)=1, a\left(n_{1}, n_{3}\right)=1$, y para el segundo artículo se tendría $a\left(n_{1}, n_{2}\right)=1$, por tanto, la centralidad de grado del autor $\mathrm{n}_{1}$ corresponde a $C_{G}\left(n_{1}\right)=1+1+1=$ 3 , esto siguiendo la ecuación 2.

Se destaca que varios autores (12) presentaron centralidad de grado igual a uno; entre ellos, el autor $n_{22}$, con un valor $C_{G}\left(n_{22}\right)=1$, que refleja que solo publicó un artículo y con solo un coautor. Así mismo, 62 autores presentaron una centralidad de grado de 2. La mayor centralidad de grado se presentó con el autor $n_{92}$, quien arrojó un $C_{G}\left(n_{92}\right)=55$.

Una forma de agilizar este proceso es con ayuda del programa Microsoft Excel, realizando las posibles combinaciones de los autores de cada uno de los artículos, relacionándolos 2 a 2 , y posteriormente contar el número de combinaciones en las que aparece cada uno de los autores.

Así, entonces, realizando este procedimiento para cada uno de los $\mathrm{N}=296$ autores, se registró un total de 2198 vínculos en toda la red, de esta forma:

$$
D=\frac{2198}{296(296-1)}=0,025
$$

De acuerdo con lo indicado por Wasserman y Faust (1994), con una densidad de red equivalente a 0,025 puede indicarse que existen muy pocas relaciones entre los autores presentes en la red. Es decir, que los integrantes de los grupos del área de Biología no registran mayor colaboración entre ellos.

El mayor grado de centralidad de un autor en la red que se obtuvo fue $C_{G}\left(n_{202}\right)=55$, por lo tanto, se tiene que $C_{G}\left(n^{*}\right)$ :

$$
C_{G}=\frac{\sum_{i=1}^{296}\left[C_{G}\left(n^{*}\right)-C_{G}\left(n_{i}\right)\right]}{N^{2}-3 N+2}
$$

$$
\begin{aligned}
& =\frac{(55-0) * 1+(55-1) * 12+(55-2) * 62+\cdots+(55-54) * 1+(55-55) * 1}{296^{2}-3(296)+2} \\
& C_{G}=\frac{14082}{86730}=0,162
\end{aligned}
$$


Esta baja centralidad de grado para toda la red refleja, como lo indica Polanco (2006), que no existe un autor que centralice la producción en el área de Biología, es decir, que se haya relacionado con todos los demás autores o con la mayoría de ellos. Como se abordará más adelante, la centralidad de grado es mayor al evaluarla en el interior de los grupos.

Adicionalmente, la red visualiza algunos tipos de nodos descritos por Polanco (2006), entre ellos la ocurrencia de nodos Aislados, que, como su mismo nombre lo indica, no se conectan con otros actores; nodos Transmisores, básicamente generados y liderados por el autor más productivo, y nodos Receptores, que corresponden a quienes reciben el acompañamiento y el trabajo de los Transmisores.

\subsubsection{Indicadores de Red para cada uno de los grupos de investigación}

Realizando un análisis análogo al contemplado para toda la red, se presentan a continuación resultados de los indicadores de tamaño (T), densidad (D) y centralidad de grado $\left(\mathrm{C}_{\mathrm{G}}\right)$ para cada uno de los 8 grupos de investigación del área de Biología de la UPTC contemplados en el estudio. En la tabla 1 se resume los valores correspondientes.

Tabla 1. Indicadores de red para cada grupo de investigación

\begin{tabular}{|c|c|c|c|c|c|}
\hline $\begin{array}{c}\text { Grupo de } \\
\text { investigación }\end{array}$ & $\begin{array}{c}\text { Líneas de } \\
\text { investigación según GrupLac }\end{array}$ & $\begin{array}{l}\text { No de } \\
\text { artículos }\end{array}$ & $T$ & $D$ & $C_{G}$ \\
\hline $\begin{array}{l}\text { BIOPLASMA } \\
\text { G1 }\end{array}$ & $\begin{array}{l}\text { 1. Producción vegetal } \\
\text { 2. Silvicultura, explotación forestal y servicios } \\
\text { relacionados }\end{array}$ & 14 & 19 & 0,240 & 1,160 \\
\hline $\begin{array}{l}\text { EBAC } \\
\text { G2 }\end{array}$ & $\begin{array}{l}\text { 1. Ecología de insectos de alta montaña de la } \\
\text { Cordillera Oriental } \\
\text { 2. Ecología de la restauración y restauración } \\
\text { ecológica en ecosistemas alto andinos de Boyacá }\end{array}$ & 10 & 13 & 0,205 & 0,742 \\
\hline $\begin{array}{l}\text { GEBIMOL } \\
\quad \text { G3 }\end{array}$ & $\begin{array}{l}\text { 1. Biología Molecular aplicada a estudios de } \\
\text { Filogenética animal } \\
\text { 2. Biología molecular y celular aplicada al } \\
\text { campo agronómico }\end{array}$ & 7 & 15 & 0,248 & 0,621 \\
\hline $\begin{array}{l}\text { GEO } \\
\text { G4 }\end{array}$ & $\begin{array}{l}\text { 1. Biodiversidad y biogeografía } \\
\text { 2. Genética de Vertebrados Tetrápodos }\end{array}$ & 11 & 29 & 0,505 & 0,148 \\
\hline $\begin{array}{l}\text { GIBC } \\
\text { G5 }\end{array}$ & $\begin{array}{l}\text { 1. Biología Vegetal y Ecología Animal } \\
\text { 2. Etnobiología }\end{array}$ & 9 & 10 & 0,200 & 0,028 \\
\hline $\begin{array}{l}\text { SISBIO } \\
\text { G6 }\end{array}$ & $\begin{array}{l}\text { 1. Sistemática de Angiospermas } \\
\text { 2. Taxonomía y Sistemática de insectos }\end{array}$ & 76 & 119 & 0,060 & 0,413 \\
\hline $\begin{array}{l}\text { UDESA } \\
\text { G7 }\end{array}$ & $\begin{array}{l}\text { 1. Biomonitoreo de Comunidades Acuáticas y } \\
\text { Calidad Ambiental } \\
\text { 2. Taxonomía, Ecología y Procesos Productivos } \\
\text { en Plancton }\end{array}$ & 20 & 46 & 0,267 & 1,005 \\
\hline $\begin{array}{l}\text { XIUÂ } \\
\text { G8 }\end{array}$ & $\begin{array}{l}\text { 1. Ecología Acuática y Ambiental } \\
\text { 2. Ecología y Ecofisiología Vegetal }\end{array}$ & 19 & 51 & 0,093 & 0,237 \\
\hline
\end{tabular}


Respecto al tamaño de la red, se observa que G6 presenta la mayor cantidad de autores distintos que publicaron por lo menos un artículo vinculado al grupo, en el periodo de estudio ( $\mathrm{T}=119)$; además, este grupo se visualiza como la subred con mayor cantidad de nodos dentro de la red de coautoría, y cuyos diámetros son de mayor tamaño, comparados con los nodos de los otros grupos (Gráfico 2). Respecto al tamaño (T), se destacan los grupos G8 y G7, con valores de 51 y 46 respectivamente.

Comparando la densidad de cada grupo (D), esto es, la cantidad de relaciones existentes dentro de cada subred de coautoría, se observa a través de la tabla 1 que los grupos presentan diferentes valores en torno a esta medida. Se destaca que G6 y G8 son los de menor densidad, con valores de 0,06 y 0,093 , respectivamente, lo cual indicaría que en cada grupo hubo pocas relaciones entre sus integrantes. G4, presenta la mayor densidad, con un valor de 0,505. Para el cálculo de los anteriores valores se procedió con las siguientes expresiones

$$
\begin{gathered}
D(G 6)=\sum_{i=1}^{N=119} \frac{C_{G}\left(n_{i}\right)}{119(119-1)}=\frac{844}{119(119-1)}=0,0601 \\
D(G 8)=\sum_{i=1}^{N=51} \frac{C_{G}\left(n_{i}\right)}{51(51-1)}=\frac{236}{51(51-1)}=0,0925 \\
D(G 4)=\sum_{i=1}^{N=29} \frac{C_{G}\left(n_{i}\right)}{29(29-1)}=\frac{410}{29(29-1)}=0,5049
\end{gathered}
$$

En el grupo G6, se identifican varios líderes en cuanto a la producción científica, sin embargo, se observa que no hay mayor comunicación entre ellos y los demás integrantes del grupo; la comunicación visualizada y calculada a través del valor D muestra que dentro de la misma subred se generan subgrupos. El grupo G8, que también presenta bajo nivel de densidad, visibiliza, a través del gráfico 2 , que existe un investigador que tiene alta productividad y que se relaciona con varios autores del grupo, sin embargo, es notorio que no existen mayores relaciones entre los demás integrantes con el autor más productivo. Este comportamiento es diferente en el grupo $\mathrm{G} 4$, que presenta mayor densidad ( $\mathrm{D}=0,505)$, en el gráfico 2 se visualiza que hay mayor comunicación de los integrantes del grupo entre sí, es decir, se generan menos subgrupos de coautores, lo que se podría asociar a una red estrella, según lo definido por García (2013), donde la mayoría de los autores se interrelacionan con los demás.

En general, se observa que en cada grupo de investigación (subredes) los autores no mantienen vínculos constantes con los mismos coautores, lo cual hace que la red de coautoría para cada uno de los grupos sea poco densa, reflejada en densidades menores a 0,3 para el $87.5 \%$ de los grupos analizados. 
La centralidad de grado de las subredes $\left(\mathrm{C}_{\mathrm{G}}\right)$ dadas dentro de cada uno de los grupos refleja que el grupo G5 presenta un menor valor de centralidad $\mathrm{C}_{\mathrm{G}}=0,028$, seguido del grupo G4 y el grupo G8, con valores de 0,14 y 0,237 , respectivamente. Los grupos que arrojan mayor valor de centralidad de grado son el grupo G1 y el grupo G7, con valores de 1,16 y 1,01 , respectivamente.

Como se había descrito anteriormente, la centralidad de grado requiere determinar para cada grupo tanto la mayor centralidad de un autor dentro del grupo, $C_{G}\left(n^{*}\right)$, como las centralidades de los demás integrantes del grupo, $C_{G}\left(n_{i}\right)$, determinando igualmente el número de autores diferentes por grupo, $\mathrm{N}$.

Al retomar la expresión general de centralidad de grado de la red (ecuación 3), ahora para cada grupo, se obtienen los resultados de la tabla 1, siendo, por ejemplo, para el Grupo 5:

$$
C_{G 5}=\frac{\sum_{i=1}^{N=10}\left[C_{G 5}\left(n^{*}\right)-C_{G 5}\left(n_{i}\right)\right]}{N^{2}-3 N+2}
$$

Siendo $\mathrm{N}=10$ autores diferentes, con el mayor número de vínculos generados de $C_{G 5}\left(n^{*}\right)=2$, de modo que

$$
C_{G 5}=\frac{(2-0) * 1+(2-1) * 0+(2-2) * 9}{10^{2}-3(10)+2}=\frac{2}{72}=0,028
$$

De donde se observa que hay un autor que tiene centralidad cero, visto, así como un nodo aislado, y que 9 autores que tienen centralidad 2.

De forma análoga, para el Grupo 1 se tiene:

$$
C_{G 1}=\frac{\sum_{i=1}^{N=19}\left[C_{G 1}\left(n^{*}\right)-C_{G 1}\left(n_{i}\right)\right]}{N^{2}-3 N+2}
$$

Siendo $\mathrm{N}=19$ autores diferentes, con el mayor número de vínculos generados de $C_{G 1}\left(n^{*}\right)=23$, de modo que

$$
\begin{aligned}
& C_{G 1}=\frac{(23-2) * 8+(23-3) * 9+(23-16) * 1+(23-23) * 1}{19^{2}-3(19)+2}=\frac{355}{306} \\
& =1,16
\end{aligned}
$$

Representando con lo anterior que hay 8 autores que tienen centralidad 2; 9 autores con centralidad 3; un autor con centralidad 16, y un autor con centralidad 23.

Esta alta centralidad de grado, para el grupo G1, expresa, como sostiene Polanco (2006), que existen autores que tiende a centralizar la producción; para este caso, se trata de los codificados con los números $155\left(\mathrm{n}_{155}\right)$ y $170\left(\mathrm{n}_{170}\right)$, visualizados en el gráfico 2 . 


\section{Conclusiones}

La mayor productividad en cada grupo de investigación, por lo general, corresponde a la del coordinador del grupo. La colaboración entre los grupos fue muy poca, a pesar de que algunas de sus líneas de investigación son afines; son mayores sus relaciones con instituciones externas tanto nacionales como internacionales.

En la mayoría de los grupos de investigación el número de publicaciones en el periodo de estudio fue relativamente bajo, lo cual explica por qué estos no se encuentran en las categorías más altas, según el modelo de medición de Colciencias.

Mediante una red de coautoría el diámetro de cada nodo está asociado con la productividad de cada autor; la capacidad de un autor para comunicarse con más autores, la cual es visible por la cantidad de vínculos entre los nodos de la red; la comunicación asertiva y frecuente entre autores para continuas publicaciones, lo cual se refleja en el grosor de las líneas o vínculos.

En este caso de estudio fue posible identificar quiénes lideran la productividad de cada uno de los grupos de investigación y establecer que no existe colaboración entre los integrantes de los diferentes grupos de investigación de una misma disciplina. Lo anterior se reflejó en las medidas de centralidad de grado y densidad de la red de cada grupo, al obtener valores relativamente pequeños.

\section{Referencias bibliográficas}

Abbasi, A. \& Altmann, J. (2010). A Social Network System for Analyzing Publication Activities of Researchers, TEMEP Discussion Paper, South-Korea, Seoul National University.

Aguado-López, E.; Rogel-Salazar, R.; Garduño-Oropeza, G.; Becerril-García, A.; ZúñigaRoca, M. F. \& Velázquez-Álvarez, A. (2009). Patrones de colaboración científica a partir de redes de coautoría. Convergencia. Revista de Ciencias Sociales, Redalyc. $<$ http://www.redalyc.org/articulo.oa?id=10512244010>. [Consulta: 21/01/2017]

Almero Canet, A. (2011). La colaboración interinstitucional en la producción científica española en enfermería: indicadores bibliométricos y Análisis de Redes Sociales. $<$ Repositorio digital CSIC. http://digital.csic.es/handle/10261/44837>. [Consulta: 21/01/2017]

Bordons, M. \& Zulueta A. (1999). Evaluación de la actividad científica a través de indicadores bibliométricos. Revista española de cardiología, 52 (10), 790-800. https://doi.org/10.1016/S0300-8932(99)75008-6

Colciencias: Sistema Nacional de Ciencia y Tecnología (1992). Convocatoria a la creatividad. Bogotá. Tercer Mundo Editores, 329 . <http://repositorio.colciencias.gov. co:8081/jspui/handle/11146/55>.

Corral Marfil, J. A., \& Cànoves, G. (2014). La colaboración científica en turismo: grupos y redes de investigación en Cataluña. Documentos de Análisis Geográfica, 1 (60).

Fonseca, S.; Ramírez, D.; Castellanos, O. (mayo de 2017). Gestión del tejido social en grupos de investigación y desarrollo a partir de análisis introspectivo de indicadores cienciométricos. En: XI Congreso Internacional de la Academia de Ciencias Administrativas (Acacia). XI Congreso Anual de Investigación, llevado a cabo en Guadalajara, Jalisco.

Freeman, L. C. (2000). La centralidad. El problema en las redes sociales. De la centralidad. Clarificación conceptual. 
García, A. (2013). Las redes de colaboración científica y su efecto en la productividad. Un análisis bibliométrico. Investigación Bibliotecológica: Archivonomía, Bibliotecología e Información, 59 (27), 159-175.

González Alcaide, G.; Gómez Ferri, J. (2014). La colaboración científica: principales líneas de investigación y retos de futuro. Revista Española de Documentación Científica, 37(4): e062. doi: http://dx.doi.org/10.3989/redc.2014.4.1186

Hou, Haiyan, Kretschmer, Hildrun, \& Liu, Z. (2008). The structure of scientific collaboration networks in Scientometrics. Scientometrics, 2 (75), 189-202.

Huamaní, C.; Mayta-Tristán, P. (2010). Producción científica peruana en medicina y redes de colaboración, análisis del Science Citation Index 2000-2009. Rev Peru Med Exp Salud Pública, 3 (27), 315-25.

Lassi, M. \& Sonnenwald, D. H. (2010). Identifying factors that may impact the adoption and use of a social science collaboratory: a synthesis of previous research. In The Seventh International Conference on Conceptions of Library and Information Science (CoLIS)- "Unity in diversity", 3, (15).

Leahey, E. (2016). From sole investigator to team scientist: trends in the practice and study of research collaboration. Annual Review of Sociology, 42.

Lee, Bangrae; Kwon, Ohjin, \& Kim, Han-joon. (2011). Identification of dependency patterns in research collaboration environments through cluster analysis. Journal of Information Science, 1 (31), 67-85.

Lewison, G. (1991). The advantages of dual nationality. New Scientist, 130, 50-51.

Luo, J.-D. (2005). Social network structure and performance of improvement teams. International Journal of Business Performance Management, 2 (7), 208-223.

Molina, M. M. (2014). Análisis del dominio científico colombiano: una visión macro a partir de datos Sciverse Scopus, 2003-2010. Tesis doctoral, Universidad de Granada.

Narin, F.; Stevens, K.; Whitlow, E. (1991). Scientific co-operation in Europe and the citation of multinationally authored papers. Scientometrics, 3 (21), 313-323.

Navarro Sánchez, L. \& Salazar Fernández, J. (2007). Análisis de redes sociales aplicado a redes de investigación en ciencia y tecnología. Sínt. Tecnol, 2 (3), 69-86.

Perianes-Rodríguez, A. (2007). Análisis y visualización de redes de colaboración científica: grupos de investigación en la Universidad Carlos III de Madrid (ISI, Web of Science, 1990-2004). Disponible en: https:/e-archivo.uc3m.es/bitstream/handle/10016/ 11428/antonio_perianes_tesis.pdf. [Consulta: 22/01/2017].

Ospina Rúa, DN. (2009). Caracterización de la producción científica y visibilidad de los investigadores de la Universidad Nacional de Colombia, sede Medellín, en la ISI Web of Science (1990-2007). Medellín: Universidad Nacional de Colombia. Disponible en: http://www.bdigital.unal.edu.co/2463/1/32322020.2009.pdf [Consulta 18/11/2016]

Polanco, X. (2006). Análisis de redes: introducción. In: Mario Albornoz \& Claudio Alfaraz. Redes de conocimiento: Construcción, dinámica y gestión. RICYT/CYTED/UNESCO Edición, Buenos Aires, pp.77-112, ISBN-10: 987-98831-1-X ISBN-13: 978-987-988311-2. $<$ https://halshs.archivesouvertes.fr/file/index/docid/218397/filename/XP_Analisis_ de_redes_2006.pdf $>$. [Consulta: 22/01/2017].

Reagans, R., \& Zuckerman, E. W. (2001). Networks, diversity, and productivity: The social capital of corporate R\&D teams. Organization Science, 4 (2), 502-517.

Rey, R. J., Martín Sempere, M., \& Sebastián, J. (2008). Estructura y dinámica de los grupos de investigación. Arbor, 732 (184), 743-757.

Sanz Casado, E. (2000). Proyecto docente para la provisión de una plaza de Catedrático de Universidad sobre Bibliometría. Getafe: Universidad Carlos III de Madrid.

Sanz Casado, E.; Martín Moreno, C. (1997). Técnicas bibliométricas aplicadas a los estudios de usuarios. Revista General de Información y Documentación, 2 (7), 41- 68.

Schultz-Jones, B. (2009). Examining information behavior through social networks: An interdisciplinary review. Journal of Documentation, 4 (65), 592-631. 
Scopus (2014). Scimago journal and Country Rank ® . http://www.scimagojr.com/ journalrank.php. [Consulta: 15/03/2014].

Sharma, M., \& Urs, S. H. (2008). Network of Scholarship: Uncovering the Structure of Digital Library Author Community. Digital Libraries: Universal and Ubiquitous Access to Information, Proceedings, 5362, 363-366.

Sorensen, A. A., Seary, A., \& Riopelle, K. (2010). Alzheimer's Disease Research: A COIN Study Using Co-authorship Network Analytics. Procedia Social and Behavioral Sciences, 4 (2), 6582-6586.

Spinak, E. (1996). Diccionario enciclopédico de Bibliometría, Cienciometría e Informetría. Caracas (Venezuela): Unesco-CII/II.

Tichy, N. M., Tushman, M. L., \& Fombrun, C. (1979). Social network analysis for organizations. Academy of Management Review, 4 (4), 507-519.

Tijssen, R. J. y Van Raan, A. F. J. (1994). Mapping changes in Science and Technology. Bibliometric co-occurrence analysis of the R\&D literature. Evaluation Review, 1 (18), 98-115.

Troyano, R., Martínez Gasca, R., González Abril, L., \& Velasco Morente, F. (2005). Análisis de redes sociales mediante diagramas estratégicos y diagramas estructurales. Redes: revista hispana para el análisis de redes sociales, 8 .

Trujillo Cancino, J. L., Guzmán Sánchez, M. V. (2013). Los mapas bibliométricos o mapas de la ciencia: una herramienta útil para desarrollar estudios métricos de información. Biblioteca Universitaria, 2 (16), 95-108.

Van der Leij, M. J., \& Goyal, S. (2011). Strong ties in a small world. Review of Network Economics, 2 (10), 1.

Vargas, F. A., \& Castellanos Domínguez, Ó. F. (2005). Vigilancia como herramientas de innovación y desarrollo tecnológico. Caso de aplicación: sector de empaques plásticos flexibles. Ingeniería e Investigación, 2 (25), 32-41.

Velden, T., Haque, A. U., \& Lagoze, C. (2010). A new approach to analyzing patterns of collaboration in co-authorship networks: mesoscopic analysis and interpretation. Scientometrics, 1 (85), 219-242.

Wasserman, S., \& Faust, K. (1994). Social network analysis: Methods and applications (vol. 8). Cambridge University Press. 